Supporting information for:

\title{
Rhodium-Catalyzed Asymmetric Hydrogenation of Functionalized Olefins Using Monodentate Spiro Phosphoramidite Ligands
}

Yu Fu, ${ }^{\dagger}$ Xun-Xiang Guo, ${ }^{\ddagger}$ Shou-Fei Zhu, ${ }^{\dagger}$ Ai-Guo Hu, Jian-Hua Xie, ${ }^{\dagger}$ Qi-Lin Zhou ${ }^{*, \dagger}$

State Key Laboratory and Institute of Elemento-organic Chemistry, Nankai University, Tianjin 300071, China, and Institute of Fine Chemicals, East China University of Science and Technology, Shanghai 200237, China

\section{General}

All reactions and manipulations were performed in an argon-filled glovebox or using standard Schlenk techniques. ${ }^{1} \mathrm{H},{ }^{13} \mathrm{C}$ and ${ }^{31} \mathrm{P}$ NMR spectra were recorded on Varian Mercury Vx-300 spectrometer. Chemical shifts were reported in ppm downfield from internal $\mathrm{Si}\left(\mathrm{CH}_{3}\right)_{4}$ and external $85 \% \mathrm{H}_{3} \mathrm{PO}_{4}$, respectively. X-Ray crystallographic analyses were performed on Bruker Smart 1000 CCD spectrometer. GC analyses were performed using Hewlett Packard Model HP 6890 Series. HPLC analyses were performed using Hewlett Packard Model HP 1100 Series.

Anhydrous toluene, $n$-hexane and THF were distilled from sodium benzophenone ketyl. EtOAc and $\mathrm{CH}_{2} \mathrm{Cl}_{2}$ were distilled from calcium hydride. Acetone was distilled from $\mathrm{P}_{2} \mathrm{O}_{5}$. $\mathrm{MeOH}$ was distilled from magnesium. Hydrogen gas (99.999\%) was purchased from Boc Gas Inc., Tianjin. $\mathrm{Rh}(\mathrm{COD})_{2} \mathrm{BF}_{4}$ and other Rh-precatalyst, ${ }^{\mathrm{i}}$ Enamide, ${ }^{\mathrm{ii}} \alpha-{ }^{\mathrm{iii}}$ and $\beta$-dehydroamino acid derivatives $^{\mathrm{iv}}$ were prepared according to literature methods.

General procedure for asymmetric hydrogenation of $\alpha$-dehydroamino acid derivatives 
To a Schlenk tube equipped with a septum and a stirring bar, in which 5 umol of [Rh], $10.5 \mu \mathrm{mol}$ of ligand and $0.5 \mathrm{mmol}$ of $\alpha$-dehydroamino acid derivative were added. After three vacuum/hydrogen cycles, $5 \mathrm{~mL}$ of solvent was added by a syringe and the reaction mixture was left stirring at room temperature under ambient $\mathrm{H}_{2}$ pressure. After the reaction was complete, the resulting mixture was filtered through a short silica column, concentrated and submitted to analysis and ee determination.

\section{General procedure for asymmetric hydrogenation of aryl enamides}

A reaction tube equipped with a stirring bar, in which $5 \mu \mathrm{mol}$ of $[\mathrm{Rh}], 10.5 \mu \mathrm{mol}$ of ligand and $0.5 \mathrm{mmol}$ of enamide were added under an inert atmosphere, was put into an autoclave. After $5 \mathrm{~mL} \mathrm{CH}_{2} \mathrm{Cl}_{2}$ added, the inert atmosphere was replaced by three hydrogen/release cycles and the reaction mixture was left stirring at request temperature and hydrogen pressure. The resulting mixture was filtered through a short silica column, concentrated and submitted to analysis and ee determination.

\section{General procedure for asymmetric hydrogenation of $\beta$-dehydroamino esters}

A reaction tube equipped with a stirring bar, in which $5 \mu \mathrm{mol}$ of $[\mathrm{Rh}], 10.5 \mu \mathrm{mol}$ of ligand and $0.5 \mathrm{mmol}$ of $\beta$-dehydroamino ester were added under an inert atmosphere, was put into an autoclave. After $5 \mathrm{~mL} \mathrm{CH}_{2} \mathrm{Cl}_{2}$ added, the inert atmosphere was replaced by three hydrogen/release cycles and the reaction mixture was left stirring at request temperature and hydrogen pressure. The resulting mixture was filtered through a short silica column, concentrated and submitted to analysis and ee determination. 
Table 8. Crystal data and structure refinement for [Rh(COD)((S)-SIPHOS-Me)](OH)

$$
\begin{gathered}
\text { Empirical formula } \\
\text { Formula weight } \\
\text { Temperature } \\
\text { Wavelength } \\
\text { Crystal system } \\
\text { Space group }
\end{gathered}
$$

Unit cell dimensions

Volume

Z, Calculated density

Absorption coefficient

$$
\mathrm{F}(000)
$$

Crystal size

Theta range for data collection

Limiting indices

Reflections collected / unique

Completeness to theta $=25.03$

Max. and min. transmission

Refinement method

Data / restraints / parameters

Goodness-of-fit on $\mathrm{F}^{2}$

Final $\mathrm{R}$ indices [l>2sigma(I)]

$\mathrm{R}$ indices (all data)

Absolute structure parameter

Largest diff. peak and hole

Absorption correction

$$
\mathrm{C}_{47} \mathrm{H}_{55} \mathrm{Cl}_{2} \mathrm{~N}_{2} \mathrm{O}_{5} \mathrm{P}_{2} \mathrm{Rh}
$$

963.68

293(2) K

$0.71073 \AA$

Tetragonal

P4(1)2(1)2

$$
\begin{gathered}
\mathrm{a}=10.495(2) \AA, \mathrm{b}=10.495(2) \AA, c=42.528(12) \AA \\
\alpha=90 \mathrm{deg}, \beta=90 \mathrm{deg}, \gamma=90 \mathrm{deg} \\
4684.1(19) \AA^{3} \\
4,1.367 \mathrm{Mg} / \mathrm{m}^{3} \\
0.593 \mathrm{~mm}^{-1} \\
2000 \\
0.25 \times 0.20 \times 0.20 \mathrm{~mm} \\
2.74 \text { to } 25.03 \mathrm{deg} . \\
-12<=\mathrm{h}<=12,-12<=\mathrm{k}<=12,-36<=\mathrm{l}<=50 \\
19274 / 4099[\mathrm{R}(\text { int })=0.0416] \\
98.3 \% \mathrm{~b} \\
0.8906 \text { and } 0.8659
\end{gathered}
$$

Full-matrix least-squares on $\mathrm{F}^{2}$

4099 / 0 / 294

$$
1.115
$$

$$
\begin{aligned}
& \mathrm{R} 1=0.0519, \mathrm{wR} 2=0.1425 \\
& \mathrm{R} 1=0.0560, \mathrm{wR} 2=0.1464
\end{aligned}
$$

$$
0.03(7)
$$

$$
0.884 \text { and }-0.630 \text { e. } \AA^{-3}
$$

Semi-empirical from equivalents 
Table 2. Selected bond length and angle for $\left[\mathrm{Rh}(\mathrm{COD})((\mathrm{S})-\mathrm{SIPHOS}-\mathrm{Me})_{2}\right](\mathrm{OH})$

\begin{tabular}{cccc}
\hline $\mathrm{Rh}(1)-\mathrm{P}(1)$ & $2.2864(16)$ & $\mathrm{P}(1)-\mathrm{N}(1)$ & $1.635(6)$ \\
$\mathrm{P}(1)-\mathrm{O}(2)$ & $1.626(5)$ & $\mathrm{N}(1)-\mathrm{C}(18)$ & $1.448(10)$ \\
$\mathrm{P}(1)-\mathrm{O}(1)$ & $1.626(5)$ & $\mathrm{N}(1)-\mathrm{C}(19)$ & $1.504(10)$ \\
$\mathrm{P}(1)-\mathrm{Rh}(1)-\mathrm{P}(1) \# 1$ & $95.66(9)$ & $\mathrm{O}(2)-\mathrm{P}(1)-\mathrm{Rh}(1)$ & $106.38(17)$ \\
$\mathrm{O}(2)-\mathrm{P}(1)-\mathrm{O}(1)$ & $102.7(2)$ & $\mathrm{O}(1)-\mathrm{P}(1)-\mathrm{Rh}(1)$ & $115.38(18)$ \\
$\mathrm{O}(2)-\mathrm{P}(1)-\mathrm{N}(1)$ & $109.2(3)$ & $\mathrm{N}(1)-\mathrm{P}(1)-\mathrm{Rh}(1)$ & $123.7(2)$ \\
$\mathrm{O}(1)-\mathrm{P}(1)-\mathrm{N}(1)$ & $97.5(3)$ & & \\
$\mathrm{P}(1) \# 1-\mathrm{Rh}(1)-\mathrm{P}(1)-\mathrm{O}(1)$ & $89.2(2)$ & $\mathrm{Rh}(1)-\mathrm{P}(1)-\mathrm{O}(2)-\mathrm{C}(16)$ & $174.8(4)$ \\
$\mathrm{P}(1) \# 1-\mathrm{Rh}(1)-\mathrm{P}(1)-\mathrm{N}(1)$ & $-30.1(3)$ & $\mathrm{O}(2)-\mathrm{P}(1)-\mathrm{N}(1)-\mathrm{C}(18)$ & $84.3(7)$ \\
$\mathrm{O}(2)-\mathrm{P}(1)-\mathrm{O}(1)-\mathrm{C}(2)$ & $-58.0(5)$ & $\mathrm{O}(1)-\mathrm{P}(1)-\mathrm{N}(1)-\mathrm{C}(18)$ & $-169.4(6)$ \\
$\mathrm{N}(1)-\mathrm{P}(1)-\mathrm{O}(1)-\mathrm{C}(2)$ & $-169.7(5)$ & $\mathrm{Rh}(1)-\mathrm{P}(1)-\mathrm{N}(1)-\mathrm{C}(18)$ & $-42.1(8)$ \\
$\mathrm{Rh}(1)-\mathrm{P}(1)-\mathrm{O}(1)-\mathrm{C}(2)$ & $57.3(5)$ & $\mathrm{O}(2)-\mathrm{P}(1)-\mathrm{N}(1)-\mathrm{C}(19)$ & $-80.6(8)$ \\
$\mathrm{O}(1)-\mathrm{P}(1)-\mathrm{O}(2)-\mathrm{C}(16)$ & $-63.6(5)$ & $\mathrm{O}(1)-\mathrm{P}(1)-\mathrm{N}(1)-\mathrm{C}(19)$ & $25.7(8)$ \\
\hline
\end{tabular}

\section{References}

i Schlenk, T. G.; Downes, J. M.; Milne, C. R. C.; Mackenzie, P. B.; Boucher, H.; Whelan, J.; Bosnich, B. Inorg. Chem. 1985, 24, 2334.

ii Burk, M. J.; Casy, G.; Johnson, N. B. J. Org. Chem. 1998, 63, 6084.

iii Herbst, R. M.; Shemin, D. Org. Synth. Coll. 1943, 2, 1.

iv (a) Lubell, W. D.; Kitamura, M.; Noyori, R. Tetrahedron: Asymmetry 1991, 2, 543. (b) Krapacho, A. P.; Diamanti, J.; Zayen, C.; Binghan, R. Org. Synth. 1973, 5, 198. 